\title{
Audiological testing for ototoxicity monitoring in adults with tuberculosis in state hospitals in Gauteng, South Africa
}

\author{
Katijah Khoza-Shangase ${ }^{a *}$ and Megan Stirk ${ }^{a}$ \\ aDepartment of Speech, Pathology and Audiology, School of Human and Community Development, University of the Witwatersrand, \\ Johannesburg, South Africa \\ *Corresponding author, email: katijah.khoza@wits.ac.za
}

Background: Ototoxicity monitoring during treatment of medical conditions, where the medications used are known to be toxic to the ear, is an important scope of practice of the audiologist; and, is an important clinical service aimed at enhancing patients' quality of life.

Aim: The aim of the current study was to determine audiological testing practices for ototoxicity monitoring in adults with tuberculosis in State Hospitals in Gauteng, South Africa. Specific objectives included establishing if ototoxicity (cochleotoxicity) monitoring occurs; determining the timing as well as frequency of monitoring; and, establishing what management protocols are followed once ototoxicity is established.

Methodology: A retrospective data record review design was adopted where hospital records from tuberculosis treatment units were reviewed. A total of 191 participant files comprised the research sample. Data obtained was analysed using descriptive statistics.

Results: Findings indicated that out of 5 hospitals recruited, only one had an ototoxicity monitoring programme in place. At this programme only $66 \%$ of participants were enrolled in an ototoxicity monitoring program in which baseline audiological measures were only conducted one month post treatment in $41 \%$ of the samples monitored. The majority of those monitored had repeat measures conducted once monthly. No clear and systematic medical management of ototoxicity, once identified, was found in the current sample. A small number of participants' medication was adjusted as an intervention measure.

Conclusion: Findings demonstrate the need for systematic and integrated ototoxicity monitoring programs in tuberculosis treatment centres in South Africa; as well as highlight the need for the development of evidence-based management protocols for ototoxicity within state hospitals in this South African context.

Key Words: audiological, audiologist, hearing, management, medical, protocols

\section{Introduction}

Tuberculosis (TB) is an ever growing epidemic within South Africa and forms part of one of the greatest post-apartheid health hindrances. ${ }^{1}$ The World Health Organization (WHO) estimates that the TB incidence rate within South Africa and Swaziland is over 1000 cases per 100000 people; $^{2}$ and, further estimates that within South Africa at least 1 in every 100 people develops TB each year. ${ }^{2}$ The large numbers of people treated for TB within South Africa raises many implications for the audiologist because many hospitals within developing countries, including South Africa, still make use of aminoglycosides for TB treatment due to their low cost and broad antimicrobial spectrum coverage. ${ }^{3}$ The established and well documented ototoxic nature of TB treatment highlights the importance of audiologists' involvement in the standard management plans for TB. However, universally accepted documented protocols for ototoxicity monitoring within the South African state hospitals still do not exist.

TB is an ever growing health problem worldwide. ${ }^{2}$ In 2002, the World Health Organization (WHO) estimated 8.6 million new cases of TB being reported and 1.3 million deaths due to TB. In developing countries, TB and human immunodeficiency virus (HIV) often co-occur, therefore highly active antiretroviral therapy (HAART) and ototoxic TB drugs are often given simultaneously, which further increases the potential for ototoxicity. ${ }^{3}$
The American Speech-Language-Hearing Association has constructed guidelines for audiological management. These guidelines dictate that individuals that are treated with ototoxic drugs should undergo baseline evaluation and monitoring that is based on the particular drug used. ${ }^{3}$ This practice might be common in developed countries; however, little is known regarding ototoxicity monitoring within the South African context.

According to Harris et al., ${ }^{3}$ there exist a number of obstacles that developing countries may face when attempting to implement ototoxicity monitoring. These include financial pressure and competing budgets within the healthcare systems, limited staff as well as few or no services available at small health care facilities. Due to these challenges, many of the audiological monitoring protocols that are used internationally cannot be easily and automatically implemented within the healthcare system of developing countries. ${ }^{3}$

Hearing loss has often been described as an 'invisible' condition; however, the effects it may have are far from invisible. A hearing loss may impact everyday communication, such as in the work place, at home and in society. ${ }^{4}$ Even though a hearing impairment may not be classified as a life threatening condition, it does severely affect an individual's quality of life; hence, the importance of early identification and management of such an impairment. 
Ototoxicity can be described as damage to the inner ear that is caused by a toxin. ${ }^{6}$ These toxins can be a number of different substances but are mainly medications that are used to treat life threatening illnesses. These ototoxic substances can damage the cochlear, vestibular system or the auditory nerve. ${ }^{6}$ The damage to the cochlear is the focus of the current study. According to Valente et al., ${ }^{7}$ there are a variety of medications that have been known to cause hearing loss. The main types of medication include aminoglycosides, antibiotics, loop diuretics and antineoplastic agents. Multi-drug resistant TB (MDR-TB) can be defined as TB with a resistance to both isoniazid and rifampicin, and is treated with injectable aminoglycosides, such as kanamycin and amikacin. There is, therefore, a need for prolonged treatment of MDR-TB for up to 18-24 months with aminoglycosides. ${ }^{3}$

Many symptoms that may be related to ototoxicity include hearing loss, tinnitus and balance difficulties. ${ }^{7}$ According to Konrad-Martin et al., ${ }^{8}$ around four million people in the United States are annually at risk of hearing loss because they are prescribed aminoglycoside antibiotics.

According to Campbell, ${ }^{9}$ a basic audiological assessment forms an integral part of most ototoxicity monitoring protocols. Audiological assessments that are used to monitor ototoxicity have two main purposes. These include detecting changes in hearing within the frequencies that affect speech in order to minimize the negative effect a hearing loss may have on communication. If changes are detected, alternative medication can be considered by the physician. ${ }^{9}$ The second reason for audiological monitoring is to assist the individual and the individual's family with the consequences of a hearing loss. This may include counselling, maintaining communication, introducing communication strategies and possibly providing amplification post-treatment. ${ }^{9}$

According to the American Speech-Language Hearing Association (ASHA), ${ }^{10}$ ototoxicity monitoring is the ongoing assessment of the peripheral and central auditory system functioning that determines the effects of toxic agents on the auditory system. This assessment should be performed before, during and after administration of ototoxic drugs, such as those that are used for TB, HIV and cancer treatments.

When analysing appropriate protocols there are a number of key procedures that need to be taken into account, which include pure tone audiometry, otoacoustic emissions (OAEs), and auditory brainstem responses (ABRs). In order to detect ototoxicity changes, procedures need to be sensitive to ototoxic damage and reliable over a long period of time. Detecting changes in pure tone audiometry using ultra-high frequency audiometry (up to $18 \mathrm{kHz}$ ) may be an effective indicator for a hearing loss due to drug exposure. ${ }^{8}$ Distortion product otoacoustic emissions (DPOAE) testing has also been shown to be the most reliable test as this measures early cochlear damage specifically the outer hair cells.

A study conducted by Tange et al., ${ }^{11}$ reviewed the importance of monitoring auditory function at frequencies above $8000 \mathrm{~Hz}$ to $20000 \mathrm{~Hz}$ in patients with cancer. Results showed that more than $50 \%$ of individuals presented with an irreversible hearing loss, which was distinctive in the high frequencies. This study, therefore, shows how the loss is most prevalent in the high frequencies and highlights the importance of testing of high frequencies during ototoxicity monitoring.
According to Harris et al., ${ }^{3}$ ototoxicity monitoring for patients that receive aminoglycosides should be performed once or twice a week. This protocol should include monitoring post-treatment as hearing loss may only occur up to six months from post treatment or exposure. ${ }^{3}$ Studies indicate that ototoxicity can occur as early as $72 \mathrm{~h}$ after aminoglycoside administration; thus, baseline evaluations should take place within $72 \mathrm{~h}$ of aminoglycoside administration. A subsequent evaluation should be completed within $24 \mathrm{~h}$ of the initial baseline. ${ }^{8}$

Audiological services are limited, or virtually non-existent, in many developing countries; and, therefore, hearing loss often goes undetected. ${ }^{3}$ Even if audiological monitoring is implemented, due to the lack of recourse, rehabilitation services are often not provided, which leads to a questionable value of monitoring. According to Harris et al., ${ }^{3}$ there are no universally accepted protocols for ototoxicity monitoring. Therefore, it is important to be able to identify existing ototoxicity monitoring protocols in hospitals within South Africa as well as factors which might influence implementation of such protocols.

Sturdy et al., ${ }^{12}$ conducted a retrospective study on the treatment and monitoring strategies of 50 patients initiating injectable antimicrobials for MDR-TB treatment from 2004 to 2009. This study found that $42 \%$ of patients received baseline audiological screening within two weeks of starting treatment, and 32\% had monthly audiograms. Khoza-Shangase and Jina ${ }^{13}$ conducted a study that focused on ototoxicity monitoring in the general medical practice within Gauteng, South Africa, and found that only $25 \%$ of general practitioners requested audiological assessment for individuals that are prescribed ototoxic medication. General practitioners reported that some of the reasons for limited referrals include the feasibility of referrals due to the ever increasing number of ototoxic drugs produced, as well as the fact that many practitioners do not have access to audiological services as these are not provided within every healthcare facility.

Literature indicates that there are a number of procedures that need to be implemented in order to ensure effective and efficient ototoxicity monitoring; however, most of this evidence comes from international studies which are vastly different to the South African context. This therefore demonstrates a lack of evidence within the South African context which provides the rationale for the current study. The current study aimed to determine and explore audiological testing practices and factors influencing ototoxicity monitoring in adults with tuberculosis at state hospitals in Gauteng, South Africa by exploring if ototoxicity monitoring happens; determining the timing as well as frequency of monitoring; establishing which personnel are involved; and, determining what management protocols are followed. Within the scope of this study, it was hoped that existing ototoxicity monitoring protocols that may be unique to South Africa would be identified and factors influencing ototoxicity monitoring possibly established. Identification of such context specific protocols might contribute toward establishing standardised protocols for the South African context.

\section{Aim}

The aim of the current study was to determine audiological testing practices for ototoxicity monitoring in adults with tuberculosis in State Hospitals in Gauteng, South Africa. 


\section{Objectives}

Specific objectives included establishing if ototoxicity monitoring occurs; determining the timing as well as frequency of monitoring; and, determining what audiological and medical management protocols are followed once ototoxicity is established.

Prior to the study being conducted, ethical approval was obtained from the University's Human Research Ethics Committee (HREC) and the study conformed to the ethical principles and provisions of the updated Nuremberg Code of Ethics.

\section{Method}

\section{Research design}

The study adopted a retrospective hospital data record review at TB units in five state hospitals in Gauteng. Limitations of this design include the fact that there may be restrictions to databases and that important data may not be available or on record ${ }^{14}$; although, this could also be an important finding as well.

\section{Description of the sample}

Purposive sampling technique was adopted to select participant files. ${ }^{15} \mathrm{~A}$ total of five hospitals were purposively selected to be included in the study as depicted in Table 1.

Because hospitals A-D did not have ototoxicity monitoring records for review, and this was confirmed by absent audiological records in patient files, data for analysis was obtained from hospital E. Data comprised 88 males (46\%) and 103 females (54\%) with a mean age of 36.6 years (standard deviation \pm 9.53 ) (the ages ranged from 18.2 years to 71.3 years). The average age of diagnosis of TB in the current study was 36.6 years.

\section{Selection Criteria Inclusion Criteria}

- The hospitals where the participant files were reviewed needed to be classified as state hospitals.

- The hospitals where the participant's files were reviewed needed to have TB treatment units.

- Participant records needed to be diagnosed with either general TB or MDR-TB, and treated with injectable aminoglycosides.

- All participant records from the sample needed to be adults, over the age of 18 years.

- All files to be reviewed needed to be files from 2012-2014

\section{Data collection}

The researcher was responsible for collecting and reviewing the participant records. A spreadsheet was used to organise the data from the relevant case files. The following information was collected and recorded onto the spreadsheet:

- Research code number of the patient

- Gender

- Admission and discharge dates

- Was an audiological assessment done?

-What were the reported symptoms relating to ototoxicity, such as tinnitus, hearing loss and dizziness at the time of the testing?

-What protocol or audiological tests were conducted? This would include otoscopic examination, acoustic immitance, otoacoustic emissions, pure tone audiometry (including ultrahigh frequency testing)

- How often were these tests conducted?

- Audiological and/or medical management, which would include medication changes, dose changes, recommendation and evaluation of hearing aid or hearing aid fitting, etc.

\section{Data analysis}

Data obtained was analysed following a quantitative approach to data analysis; with descriptive statics forming part of the analysis strategy ${ }^{16,17}$

\section{Reliability and validity}

Firstly, the current study ensured reliability through the use of the same and consistent data collection tool. Secondly, at least thirty percent of data collected was independently rated to ensure accurate capturing. ${ }^{18}$

\section{Results}

\section{Occurrence of ototoxicity monitoring}

Of the five hospitals included in the study, only one had a programme in place; but this was also not comprehensive. In hospital E, where ototoxicity monitoring was conducted, of the total sample, a significant majority of the patients were enrolled in an ototoxicity monitoring programme $(66 \%)$; only $34 \%$ of the patients did not undergo ototoxicity monitoring. The audiological tests that were routinely performed included detailed case history, otoscopy, tympanometry, and basic pure tone audiometry.

Of the total sub-sample enrolled in an ototoxicity monitoring programme $(n=124)$, specific patient complaints related to possible ototoxicity which were reported by patients on case

Table 1: Participating hospitals' responses regarding ototoxicity programmes

\section{Hospital \\ Hospital A \\ Hospital B \\ Hospital C \\ Hospital D \\ Hospital E}

\section{Responses}

No audiological data in files: No ototoxicity monitoring programme

No audiological data in files: No ototoxicity monitoring programme

No audiological data in files: No ototoxicity monitoring programme

Hospital's TB Centre does not provide long term inpatient care and has a focus primarily on education and health promotion programmes around ototoxicity without actual audiological monitoring

342 hospital records between 2012-2014 available for review; 191 met inclusion criteria 
Table 2: Patient complaints of symptoms related to ototoxic medication and other* symptoms $(n=124)$.

\begin{tabular}{|l|c|c|c|}
\hline Symptoms & Tinnitus & Hearing loss & Other* \\
\hline Percentage of individuals (\%) & $49 \%$ & $34.4 \%$ & $35.2 \%$ \\
\hline Other* Symptoms & Discharge & Pain & Wax \\
\hline Percentage of individuals (\%) & $2.4 \%$ & $13.6 \%$ & $0.8 \%$ \\
\hline
\end{tabular}

${ }^{*}$ Other refers to symptoms experienced not as a result of ototoxicity from medication used.

Table 3: Timing of first audiological testing post initial treatment $(n=124)$

\begin{tabular}{|l|c|c|c|c|c|c|c|c|c|}
\hline $\begin{array}{l}\text { Timing } \\
\text { (weeks) }\end{array}$ & $\begin{array}{c}\text { Baseline } \\
\text { (prior to } \\
\text { treatment) }\end{array}$ & Two weeks & Three weeks & Four weeks & $\begin{array}{c}\text { Eight weeks } \\
\begin{array}{c}\text { Twelve } \\
\text { meeks } \\
\text { meeks }\end{array}\end{array}$ \\
\hline $\begin{array}{l}\text { Percentage of } \\
\text { Individuals (\%) }\end{array}$ & $0 \%$ & $10 \%$ & $2 \%$ & $41 \%$ & $19 \%$ & $11 \%$ \\
\hline
\end{tabular}

Table 4: Frequency of audiological monitoring $(n=124)$

\begin{tabular}{|l|c|c|c|c|c|c|c|}
\hline $\begin{array}{l}\text { Frequency of } \\
\text { testing }\end{array}$ & Once a week & Once a month & $\begin{array}{c}\text { Once every } \\
\text { eight Weeks }\end{array}$ & $\begin{array}{c}\text { Once every } \\
\text { twelve weeks }\end{array}$ & $\begin{array}{c}\text { Once every } \\
\text { twenty weeks } \\
\text { or more }\end{array}$ & $\begin{array}{c}\text { Post Treatment } \\
\text { audiological } \\
\text { monitoring }\end{array}$ \\
\hline $\begin{array}{l}\text { Percentage of } \\
\text { Individuals (\%) }\end{array}$ & $6 \%$ & $47 \%$ & $5 \%$ & $6 \%$ & $7 \%$ & $2 \%$ \\
\hline
\end{tabular}

history interviews were collated from patient files. These complaints are summarised in Table 2.

Tinnitus appeared to be the most common symptom that patients reported with $49 \%$ of the total sub-sample reporting it, with hearing loss being the second most common symptom, with vertigo being least reported. A further $35 \%$ of the patients also reported 'other' symptoms that are not related to ototoxic medication, as depicted in Table 2.

\section{Timing and frequency of ototoxicity monitoring}

Table 3 depicts the time when ototoxicity monitoring occurred. No patient received baseline assessment prior to the initial treatment; the majority (41\%) of the 124 patients received an initial audiological evaluation one month post initial treatment.

The frequency of audiological monitoring during TB treatment is illustrated in Table 4.
Just under half of the 124 participants (47\%) received audiological monitoring every four weeks (once a month), with the next biggest group (27\%) only undergoing baseline assessment and no repeat testing. Only $2 \%$ of participants received audiological monitoring following treatment cessation.

\section{Audiological and medical management protocols}

In addressing the specific aim of identifying recommendations or suggested medical management protocols implemented once ototoxicity was identified (depicted in Table 5), it was found that more than half of the participants $(54 \%)$ received no medical recommendations; and, the treatment continued without any interventions. Where interventions for ototoxicity were made, the most common recommendation (34\%) was medication readjustment.

Table 6 illustrates that $7 \%$ of 124 of patients were advised a full audiological evaluation and that $6 \%$ of patients were discharged

Table 5: Recommendations/suggested medical management protocols $(n=124)$.

\begin{tabular}{|l|c|c|c|c|}
\hline $\begin{array}{l}\text { Medical } \\
\text { management }\end{array}$ & $\begin{array}{c}\text { No } \\
\text { recommendations } \\
\text { (Audiological/ } \\
\text { Medical) }\end{array}$ & $\begin{array}{c}\text { No medical } \\
\text { recommendations }\end{array}$ & $\begin{array}{c}\text { Re-adjust } \\
\text { medication (dosage) }\end{array}$ & ENT referral \\
\hline $\begin{array}{l}\text { Percentage of individ- } \\
\text { uals (\%) }\end{array}$ & $9 \%$ & $54 \%$ & $34 \%$ & $1 \%$ \\
\hline
\end{tabular}

Table 6: Recommendations/suggested audiological management protocols $(n=124)$.

\begin{tabular}{|l|c|c|c|c|}
\hline $\begin{array}{l}\text { Audiological } \\
\text { management }\end{array}$ & $\begin{array}{c}\text { Discharged from } \\
\text { audiology }\end{array}$ & $\begin{array}{c}\text { Full diagnostic } \\
\text { audiogram }\end{array}$ & $\begin{array}{c}\text { Hearing aid } \\
\text { evaluation (HAE) }\end{array}$ & $\begin{array}{c}\text { Hearing aid fitting } \\
\text { Reprogram hearing } \\
\text { aid }\end{array}$ \\
\hline $\begin{array}{l}\text { Percentage of individ- } \\
\text { uals (\%) }\end{array}$ & $6 \%$ & $7 \%$ & $3 \%$ & $2 \%$ \\
\hline
\end{tabular}


from audiology. A small number of patients were assessed for (3\%) and/or received (2\%) amplification.

\section{Discussion}

Current findings regarding lack of ototoxicity monitoring programmes in four of the 5 state hospitals recruited for the study were significant. Within the health care sector that services the majority of the South African population (state hospitals) and where the majority of TB infected patients are treated, such lack of critical preventative as well as habilitative services have serious implications for the value placed on quality of life. Although the current study was only in one province, Gauteng, this is the richest and most resourced province in the country; and, therefore, this finding has significant implications. One hospital in which ototoxicity monitoring occurred, only $66 \%$ of the sample enrolled in ototoxicity monitoring. The fact that universal coverage was not found is thus of concern. It is a fact that audiological services are limited or virtually non-existent in many developing countries, and therefore hearing loss often goes undetected in a large majority of hearing impaired populations. There are not enough audiological services present for the demand in South Africa as the number of audiologists registered with the Health Professions' Council of South Africa is abysmal when compared to the population requiring the services; and, this situation is worse in state hospitals which service at least $80 \%$ of the total population. Consequently, where audiological monitoring is being implemented for ototoxicity monitoring, the lack of necessary and enough resources as well as the limited number of staff significantly impact on the provision of appropriate rehabilitation services. Where rehabilitative resources are limited, a question about the value and ethical positioning of identification of ototoxicity is always raised. This may provide an explanation with regard to the current findings; but, does not excuse the lack of audiological services for this population. Early identification of ototoxicity and patient counselling regarding signs and symptoms of ototoxicity may in fact significantly aid in improving adherence to the TB treatment, which patients may stop taking if they do not understand the relationship between the medications they are taking and ear-related symptoms. Furthermore, if patients do not receive hearing conservation strategies to minimize concomitant impact of toxins to the ear, such as presence of ototoxicity and excessive noise, the degree of hearing loss patients present with may progressively worsen, with rehabilitation costs increasing significantly. It is also noteworthy that screening and monitoring for ototoxicity can arguably be performed by trained personnel other than audiologists, with audiologists managing these programmes; and, therefore, intensive efforts toward developing such cadres of professionals could yield positive outcomes by increasing access for a number of patients.

When examining specific patient complaints related to possible ototoxicity, current findings indicated that tinnitus appeared to be the most common symptom reported by participants, followed by hearing loss and, lastly, vertigo. These findings are typical of ototoxicity-related symptoms, and are similar to those found by Khoza-Shangase et al. ${ }^{5}$ It was also noted that $32 \%$ of participants reported that these symptoms negatively impacted their activities of daily living. This reported negative impact raises important implications around counselling and education of patients undergoing TB treatment.

According to the American Academy of Audiology, ${ }_{1}^{19}$ tinnitus is the most common symptom relating to ototoxic medication; however, there are no formal tinnitus monitoring procedures. As a result, tinnitus is analysed through patient self-reports, but it has been found that individuals with life threatening illnesses often do not report tinnitus or other symptoms relating to ototoxic medication as they are often overwhelmed with other medical issues. This, therefore, indicates that the current findings of tinnitus may not be a true reflection of tinnitus experienced in this population and may in fact be an under-reporting.

As far as the examination of the timing of baseline audiological measures was concerned, the fact that the majority (41\%) of the monitored patients only underwent their first audiological assessment a month after initiation of treatment is a serious concern. Not only does this eliminate the possibility of having pre-change hearing thresholds available for later test-retest comparisons; but, it also omits the possibility of implementing early preventative medical interventions such as drug changes, dosage adjustments, and possible prescription of otoprotective agents where and when these are available. Moreover, this delayed point of audiological entry also leads to loss of opportunity for pre-treatment counselling, which has important implications for patient expectations, adherence as well as quality of life. Current findings regarding timing of baseline measures are not in line with what the literature recommends and are also not consistent with international standards.

Studies indicate that ototoxicity can occur as early as $72 \mathrm{~h}$ after aminoglycoside administration. ${ }^{8}$ Based on this evidence baseline evaluations should take place prior to or within $72 \mathrm{~h}$ of aminoglycoside administration. A subsequent evaluation should be completed within $24 \mathrm{~h}$ of the initial baseline, ${ }^{8}$ and, current findings indicate that the state hospitals in the current study did not meet this recommendation.

When examining the frequency that audiological monitoring occurs, current findings indicate that the majority of participants only received repeat audiological assessments as part of the monitoring programme once a month. Results also indicated that a small percentage of participants received audiological monitoring post treatment cessation - another international standard. These findings are consistent with those by Sturdy et al., ${ }^{12}$ where $32 \%$ of their sample underwent monthly assessments.

According to literature, audiological monitoring evaluations should be conducted once or twice a week for individuals that receive ototoxic medication. Monitoring and referrals for audiological monitoring should also be performed at any time that a patient complains about decreased hearing abilities or symptoms such as tinnitus, dizziness or aural fullness. ${ }^{8}$ Monitoring evaluations should be continued post-treatment regimens as symptoms and changes in hearing may be documented up to six months post-treatment. ${ }^{8}$

When examining specific medical interventions implemented once ototoxicity was identified, current findings indicate a glaring break in conversion of documented theory and evidence into practice. For a majority of participants, no medical intervention was implemented. For those where intervention occurred, this came in the form of medication re-adjustments for only $34 \%$ of the sample. Results are consistent with findings by Khoza-Shangase and Jina, ${ }^{13}$ who found the preventative measures that practitioners reported consisted of changing medication and counselling patients. Other medical interventions, such as dosage changes, prescription of otoprotective agents, and hearing conservation measures, 
should form part of the TB treatment team's protocol to ensure comprehensive provision of care that takes enhanced quality of life as an important treatment success indicator. These interventions are especially important since treatments cannot be stopped and hearing loss is often of secondary importance to the primary objective of sustaining life.

Early identification of hearing loss allows medical practitioners to minimize further damage as well as to prevent a hearing loss progressing to a stage when aural rehabilitation or alternative communication is warranted. ${ }^{20}$ If a hearing loss is identified early, recommendation may include changing the medication to one that is less ototoxic, stopping treatment with the ototoxic agent or altering the medication dosage. If no change in hearing has been noted in the patient, than medical practitioners may have the option of increasing the dosage while monitoring the individual's hearing. ${ }^{20}$ Therefore, communication between professionals forms an integral part of an ototoxicity monitoring programme.

\section{Conclusion}

TB is a growing epidemic in South Africa and forms part of one of the greatest health challenges that the South African Department of Health has to manage. The large numbers of people treated for TB in South Africa raises many implications for the audiologist because many hospitals within developing countries, including South Africa, still make use of aminoglycosides for TB and MDRTB treatment. Some of the other reasons advanced for the routine use of aminoglycosides are their low cost and broad antimicrobial spectrum coverage. Current findings highlight the need for more strategic and systematic planning around ototoxicity monitoring within the state hospitals where these drugs are used.

Current findings indicate a clear gap in the clinical service provision of patients with TB in this context. The implementation of ototoxicity monitoring protocols, which also include vestibulotoxicity testing, the timing and frequency of the monitoring and the interventions provided once ototoxicity is identified, do not comply with documented recommendations and also do not compare with international standards. Current findings also highlight the lack of systematic and standard monitoring protocols for TB treatment in the state hospitals.

These findings have important implications for the audiology community in terms of awareness campaigns, as well as lobbying for the establishment of audiological clinical services in all settings where ototoxic medications are prescribed as standard treatment regimens. Furthermore, South African audiologists need to develop and standardise ototoxicity monitoring clinical guidelines that are contextually relevant and locally responsive. These findings raise additional implications for the plans and policy planning and implementation of the South African Department of Health. This is particularly important when one considers the numbers of South Africans infected with TB. Where medical management is life-saving and/or life-sustaining, it is imperative that the quality of that life receives just as much focus. Management of side effects of the treatments offered, where ototoxicity is one with this population, should be part of the standard package of care, offering comprehensive care.
The current findings should be interpreted within the identified methodological limitations, which include the small sample size which was localised to one province in the country and the retrospective design nature of the study.

\section{References}

1. Karmin SSA, Churchyard GJ, Karmin QA, et al. HIV infection and tuberculosis in South Africa: an urgent need to escalate the public health response. Lancet. 2009;374:921-3

2. WorldHealthOrganization.Globaltuberculosisreport2013.WHOLibrary Cataloguing-in-Publication Data. (WHO/HTM/TB/2013.11). 2013. http://apps.who.int/iris/bitstream/10665/91355/1/9789241564656_ eng.pdf Retrieved, 26/01/2015

3. Harris T, Peer S, Fagan JJ. Audiological monitoring for ototoxic tuberculosis, human immunodeficiency virus and cancer therapies in developing world setting. J Laryngol Otol. 2012;126:548-51.

4. Tye-Murray N. Foundations of aural rehabilitation: children, adults and their family members. Delmar Cengage Learning; Clifton Park, NY; 2009.

5. Khoza-Shangase K, Mupawose A, Mlangeni NP. Ototoxic effects of tuberculosis treatments: how aware are the patients? Afr J Pharm Pharmacol. 2009;3(8):391-9.

6. Roland PS, Pawlowski KS. Ototoxicity. In: Snow JB, Vackym PA Ballenger JJ, editors. Ballenger's otorhinolaryngology: head and neck surgery. People's Medical Publishing House; Shelton, Connecticut: 2009. p. 273-278.

7. Valente M, Hosford-Dunn H, Roeser RJ. Audiology: treatment. 2nd ed.. New York, NY: Thieme Medical ; 2008.

8. Konrad-Martin D, Gordon JS, Reavis KM, et al. Audiological monitoring of patients receiving ototoxic drugs. Perspect Hear HearDisord Res Diagn. 2005;9(1):17-22

9. Campbell KCM. Audiological monitoring for ototoxicity. In: Roland JA Rutka JA, editors. Ototoxicity. BC Decker Inc, Hamilton, London; 2004. p. $153-160$.

10. American Speech-Language- Hearing Association (ASHA). Preferred practice patterns for the profession of audiology. doi: http://dx.doi.org/10.1044/policy; 2006.

11. Tange RA, Dreschler WA, Hulst RJAM. The importance of high-tone audiometry in monitoring for ototoxicity. Arch Otorhinolaryngol. 1985;242:77-81.

12. Sturdy A, Goodman A, Jose R, et al. Multidrug-resistant tuberculosis (MDR-TB) treatment in the UK: a study of injectable and toxicity in practice. J Antimicrob Chemotherapy. 2011;66:1915-82.

13. Khoza-Shangase K, Jina K. Ototoxicity monitoring in general medical practice: exploring perceprtions and practices of general practitioners about drug-induced auditory symptoms. IPP. 2013;1(3):250-9.

14. Hess DR. Retrospective studies and chart reviews. Resp Care. 2004;49(10):1171-4.

15. Gerrish K, Lacey A, editors. The research process in nursing. WileyBlackwell Publishing West Sussex, United Kingdom; 2010.

16. Babbie E, Mouton J. The practice of social research. Cape Town University Press; 2001.

17. Sim J, Wright C. Research in health care: concepts, designs and methods. Stanley Thomas, Cheltenham, United Kingdom; 2000.

18. Pitney W, Parker J. Qualitative research in physical activity and the health professionals. Library of Congress Cataloging in Publication Data, United States of America; 2009.

19. American Academy of Audiology (AAA). Position statement and clinical practice guidelines: Ototoxicity Monitoring. 10/2009, 2009. http:// audiology-web.s3.amazonaws.com/migrated/OtoMonGuidelines. pdf_539974c40999c1.58842217.pdf Retrieved 26/01/2015

20. Fausti SA, Helt WJ, Gordon JS, et al. Audiologic monitoring for ototoxicity and patient management. In: Campbell KC, editor Pharmacology and Ototoxicity for Audiologists. San Diego CA: Thompson, Delmar Learning, 2006. p. 253-284. 